\section{Photorefractive Dark and Vortex Solitons}

By Mordechai Segev, Electrical Engineering Department, Princeton University, Princeton, N.f:; Gregory Salamo, Galen Duree, and Matthew Morin, Physics Department, University of Arkansas, Fayetteville, Ark; Bruno Crosignani and Paolo Di Porto, Dipartimento di Fisica, Universito' dell'Aquila, L'Aquila, Italy; and Amnon Yaniv, California Institute of Technology, Pasadena, Calif.

patial solitons in photorefractive (PR) materials' have been the object of growing interest during the last two years. Until now, three different types of PR solitons have been investigated. One type of PR soliton stems from the nonlocal nature of the photorefractive effect, as manifested in the dependence of the perturbation in the refractive index on the transverse derivatives of the light intensity distribution. ${ }^{12}$ Solitons of this type evolve when diffraction is exactly balanced by self-scattering (two-wave mixing) of the spatial (plane wave) components of the soliton beam. The observation of photorefractive bright solitons of the first type $^{3.4}$ revealed that, unlike the Kerr-like solitons, the PR solitons may be trapped in two transverse dimensions ${ }^{5}$ (although the self-trapping effects are inherently asymmetric with respect to the two transverse dimensions) and maintain their stability. Solitons of this type exist when an external voltage is applied to the PR material, after the index gratings have been formed, but before the external field is screened by the background conductivity. Their most
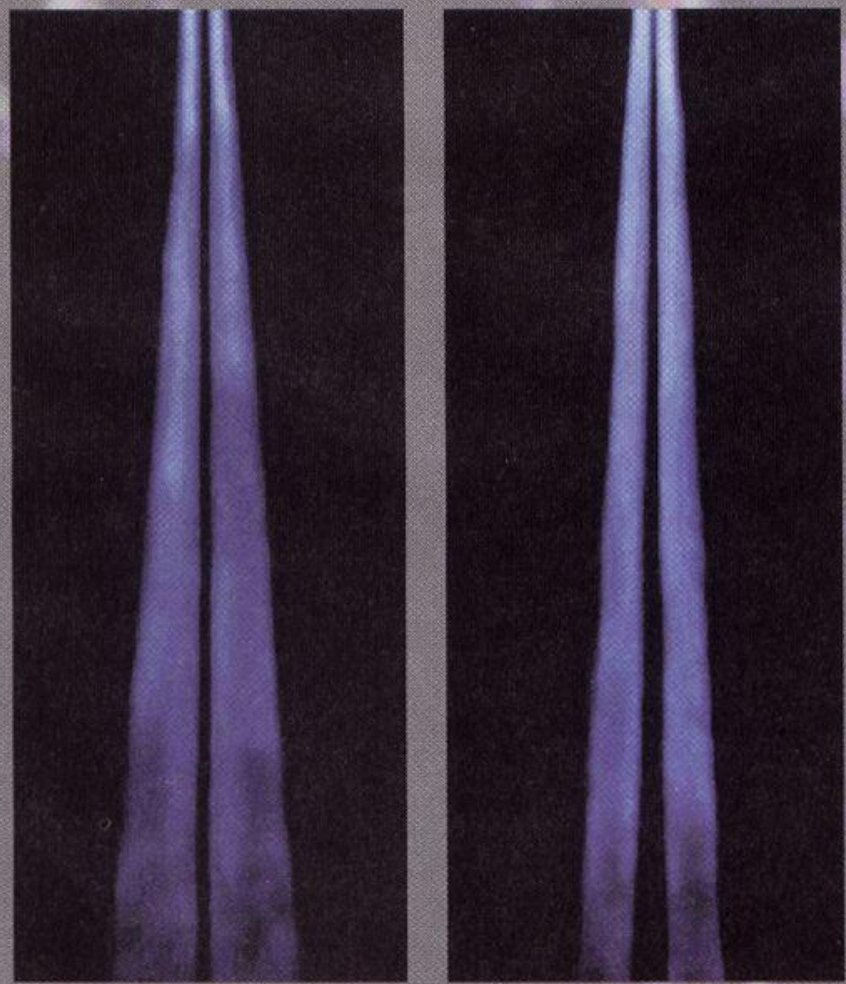

Figure 1. Top view photographs of a 21 - $\mu \mathrm{m}$ wide photorefractive dark spatial soliton (left) and a normally diffracting dark notch (right) propagating (from top to bottom) in a 5 -mm long crystal.

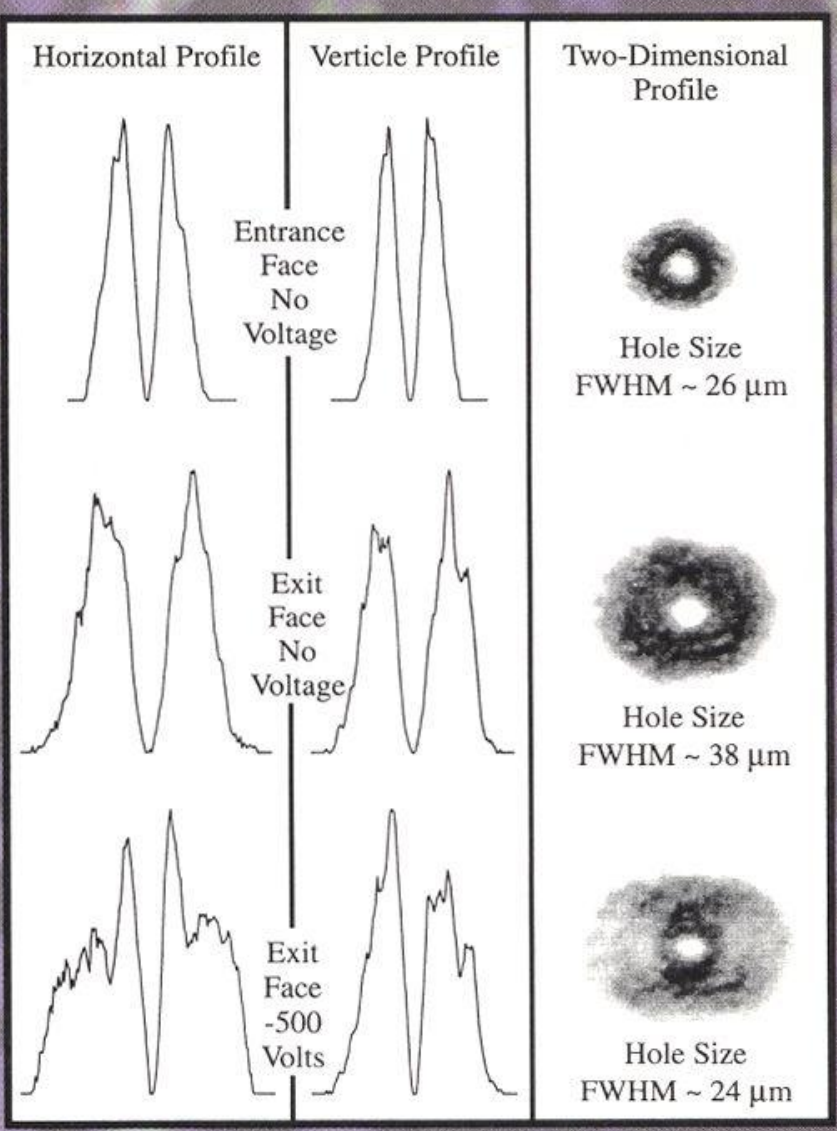

Figure 2. Two-dimensional transverse profiles and photographs of the input (upper) and diffracting optical vortices (middle) and photorefractive vortex soliton (lower).

distinct properties are (i) independence of the absolute light intensity ${ }^{1.3}$ (for intensities much larger than the dark irradiance) and (ii) the capability of trapping in two transverse dimensions. ${ }^{3.5}$ The second and third types of PR solitons, which we call screening solitons' and photovoltaic solitons, respectively, result from nonuniform screening (the former) or from photovoltaic fields (the latter). Unlike the first type of PR soliton, these two are both local effects (the index perturbation at any location is a function of the light intensity at the same location). As such, the shape and width (cross section) of these solitons depend on their intensity.

Recently, we reported on the first experimental observation of photorefractive dark solitons and vortex solitons,? both belonging to the first (nonlocal) type. To observe the planar dark solitons, we launched a dark notch with the necessary $\pi$ phase jump in its center by inserting a thin glass slide in one half of the beam. Photographs of the experimental observations are shown in Figure 1, where a $21-\mu \mathrm{m}$ wide dark soliton (non-diffracting dark notch) and a normally-diffracting (from 21 to $35 \mu \mathrm{m}$ ) notch are shown (right and left photographs, respectively). The external field required to trap the dark soliton was $-400 \mathrm{~V} / \mathrm{cm}$, applied parallel to the trapping direction. An interferometric measurement verified the $\pi$ phase jump at the center of the dark soliton. An asymmetry between the direction of trapping arises when the notch in the input beam is rotated so that the external field is parallel to the dark notch in the beam. In 
this case, the dark notch could not be trapped even at fields as high as $-1000 \mathrm{~V} / \mathrm{cm}$. An important signature of the nonlocal PR solitons is their indifference to the absolute light intensity. To verify this we varied the input power over 2 orders of magnitude, from 3 to $300 \mu \mathrm{W}$ (intensities of $0.3-30$ $\mathrm{W} / \mathrm{cm}^{2}$ ), and observed no change in the shape or the size of the dark soliton. ${ }^{9}$

To observe the photorefractive vortex solitons, it is necessary to launch a beam that bears an exp[ime] transverse phase. We used two techniques to generate the desired phase dependence at the input face of the crystal. First, we used the coherent "donut" mode of a laser and, second, we constructed the "donut" mode using a sum of two beams: one with a vertical notch and the other with a horizontal notch, with an appropriate $\pi / 2$ relative phase between them. Both methods produced a Gauss-Laguerre beam that possessed the desired phase dependence. Our results are shown in Figure 2, where the horizontal (left column) and vertical (middle) cross sections of the propagating beam are shown together with the actual photographs (right column; darker regions represent regions of higher optical intensity), under various conditions. The upper and the middle sets show the beam profiles at the entrance and the exit faces of the crystal with no applied voltage. It is apparent that the bright regions and the central, near zero intensity regions diffract in the same manner. When a negative voltage is applied (lower profiles) the central two-dimensional region (the optical vortex) becomes self-trapped, while the bright regions increase their diffraction significantly (indicative of the self-defocusing behavior). Comparison to observations of optical vortex solitons in Kerr media reveal that all the differences in the properties of both types, as discussed above for dark solitons, persist for the vortex solitons.

\section{REFERENCES}

1. M. Segev et al., "Spatial solitons in photorefractive media," Phys. Rev. Lett. 68, 923 (1992)

2. B. Crosignani et al., "Self-trapping of optical beams in photorefractive media," J. Opt. Soc. Am. B 10, 446 (1993).

3. G. Duree et al., "Observation of self-trapping of an optical beam due to the photorefractive effect," Phys. Rev. Lett. 71, 533 (1993).

4. M. Segev et al., "Dimensionality and size of photorefractive spatial solitons," Opt. \& Phot. News 4, 8 (1993).

5. G. Duree et al., "Photorefractive spatial solitons," Opt. Lett. 19, 1195 (1994).

6. M. Segev et al., "Stability of photorefractive solitons," Opt. Lett. 19, 1296 (1994).

7. M. Segev et al., "Steadystate spatial screening-solitons in photorefractive media with applied field," submitted to Phys. Rev. Lett.

8. G. C. Valley et al., "Bright and dark photovoltaic spatial solitons," submitted to Phys. Rev. Lett.

9. G. Duree $e t$ al., "Photorefractive dark spatial solitons and photorefractive vortex solitons," submitted to Phys. Rev. Lett.

\section{Directional, Enhanced Fluores- cence from Molecules on a Corrugated Optical Waveguide}

By Kevin G. Sullivan and Dennis G. Hall, The Institute of Optics, University of Rochester, Rochester, N.Y.

ptical waveguides offer many benefits to medicaldiagnostic measurements that detect the presence of thin surface layers of a target substance.' One approach involves examining the fluorescence from a layer of molecules located on or near the surface of a waveguide. If the target substance does not fluoresce, it can be tagged with fluorescent dye molecules. A properly selected waveguide system can give rise to a strong fluorescent signal.

In a recently reported set of fluorescence experiments, a metal-clad waveguide was shown to be particularly effective. ${ }^{2}$ A glass substrate was coated first with a nearly opaque layer of silver $(\mathrm{Ag})$, then with a lithium fluoride (LiF) layer of thickness $d$, and finally with a near-monolayer of Rhodamine B dye molecules (fluorescence peaks at $580 \mathrm{~nm}$. A variation on the sample geometry includes a grating formed in a layer of photoresist between the glass and the silver layer, as shown in Figure (a). In the experiments, a light-source and an apertured detector $\left(\sim 0.2^{\circ}\right.$ halfwidth), both placed on the molecule side of the sample, excited the fluorescence and collected it. The experiments measured the enhancement $\alpha$, defined as the ratio of the measured fluorescent intensity using the layered substrate to that using a glass slide as a substrate (reference sample). With no grating present and $d \sim 300 \mathrm{~nm}$, enhancements as large as $\alpha=450$ were observed. Samples with gratings produced highly directional emission with enhancements as large as $\alpha=1,100$, a striking improvement over the reference signal. Figure (b) shows a polar plot of the measured enhancement as a function of the emission angle from the sample. The pattern is symmetric about $\theta=0$; the measurement system blocks the detector in a portion of one quadrant. A linear polarizer was placed in front of the detector to record the pattern in Figure (b).

The angles for the prominent emission peaks in Figure (b) are precisely those for grating-output-coupling of the $\mathrm{TE}_{\mathrm{o}}$ (lowest-order transverse-electric) mode of the $\mathrm{Ag} / \mathrm{LiF}$

(a)

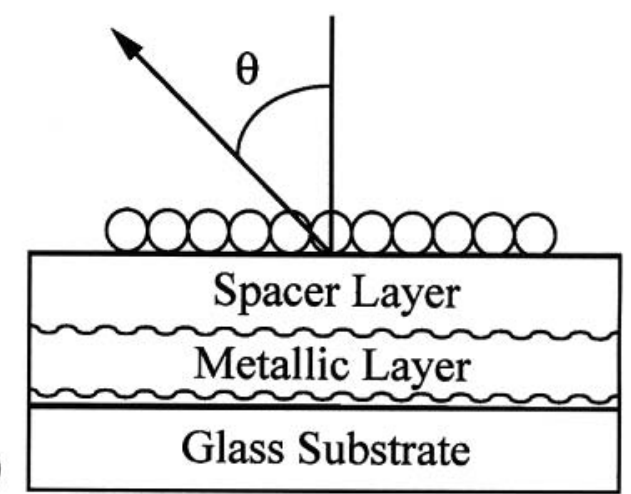

Figure (a). Basic sample geometry: a glass/photoresist-grating/metal/dielectric layered structure coated with a near-monolayer of dye molecules. 